Results Compared with normoxia, hypoxia promoted PMN survival (mean $\% \pm$ SEM apoptotic cells at $20 \mathrm{~h} ; 30.9 \pm 1.9$ vs. $59.0 \pm 1.8$ respectively, $\mathrm{p}<0.0001$ ). Both pan-PI3-K inhibitors reversed the pro-survival effect of hypoxia in a concentrationdependent manner, LY294002 $(10 \mu \mathrm{M} ; 60.3 \pm 4.0, \mathrm{p}<0.0001)$ and ZSTK474 $(10 \mu \mathrm{M} ; 58.3 \pm 2.6, \mathrm{p}<0.0001)$ without affecting the basal rate of apoptosis. This effect was not seen with the

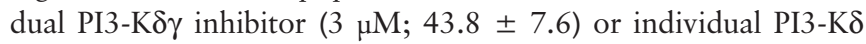
$(1 \mu \mathrm{M} ; 43.0 \pm 5.8)$ and $\gamma$ inhibitors $(3 \mu \mathrm{M} ; 34.9 \pm 4.5$ and $10 \mu \mathrm{M} ; 32.6 \pm 3.6$ ).

Conclusions Our results indicate that hypoxia-induced PMN survival is PI3-K dependent. Targeting this pathway may accelerate PMN apoptosis, resulting in resolution of inflammation.

\section{P22 ENDOPLASMIC RETICULUM STRESS MARKERS CORRELATE WITH FIBROSIS IN IDIOPATHIC PULMONARY FIBROSIS AND NON-SPECIFIC INTERSTITIAL PNEUMONIA}

${ }^{1} \mathrm{H}$ Parfrey, ${ }^{1} \mathrm{~B}$ Beardsley, ${ }^{1} \mathrm{~J}$ Knight, ${ }^{2} \mathrm{~S} J$ Marciniak, ${ }^{1} \mathrm{D}$ Rassl. ${ }^{1}$ Papworth Hospital NHSFT, Cambridge, UK, ${ }^{2}$ CIMR, University of Cambridge, Cambridge, UK

\subsection{6/thoraxjnl-2014-206260.172}

Idiopathic pulmonary fibrosis (IPF) and non-specific interstitial pneumonia (NSIP) are forms of idiopathic interstitial pneumonias that have distinct histopathological features and outcomes. It is unknown if these idiopathic interstitial pneumonias have common mechanisms of fibrosis. Endoplasmic reticulum (ER) stress has been implicated in the pathogenesis of sporadic and familial idiopathic pulmonary fibrosis. In response to endoplasmic reticulum (ER) stress, cells trigger the unfolded protein response (UPR) with upregulation of chaperones, such as BiP, and the phosphatase growth arrest and DNA damage 34 (GADD34). However, this may have profound effects on cell proliferation and survival. We hypothesised that ER stress may also be involved in the pathogenesis of NSIP.

Paraffin embedded lung biopsy sections from 4 patients with sporadic idiopathic pulmonary fibrosis (IPF) (UIP histopathology) and 4 non-specific interstitial pneumonia (NSIP) (NSIP histopathology) were evaluated for the ER stress markers BiP and GADD34 using immunohistochemistry. For each biopsy sample, six high power fields (x 200 magnification) were scored for fibrosis and inflammation as well as BiP and GADD34 using semiquantitative analysis by 2 blinded, independent investigators.

$\mathrm{BiP}$ and GADD34 were expressed in areas of fibrosis, localised to reactive type II pneumocytes and endothelial cells. No staining was detected in fibroblasts or fibroblastic foci. In sporadic IPF (UIP), levels of BiP within the epithelium correlated with fibrosis $\left(\mathrm{r}^{2} 0.56, \mathrm{p}=0.0001\right.$, Figure 1a) more than inflammation $\left(\mathrm{r}^{2} 0.38, \mathrm{p}=0.0013\right)$. In contrast, epithelial GADD34 was
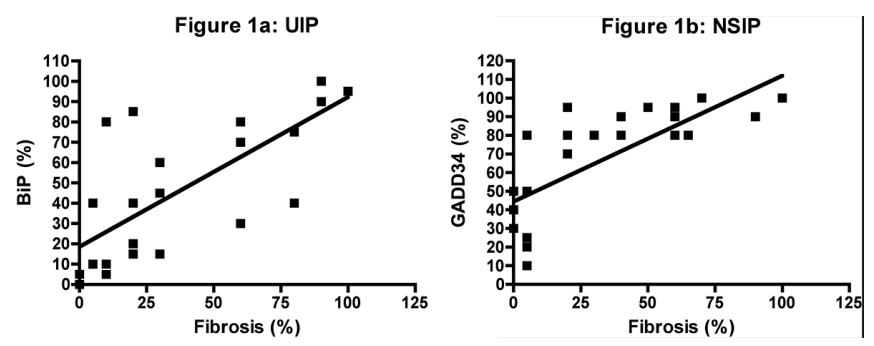

Abstract P22 Figure 1 (A) UIP, (B) NSIP more strongly associated with NSIP fibrosis $\left(\mathrm{r}^{2} \quad 0.56\right.$, $p<0.0001$, Figure 1b). There was no association between the ER stress markers and inflammation in NSIP.

These data suggest that ER stress and the unfolded protein response are features of NSIP as well as IPF and may play a role in determining the severity of the fibrotic response.

\section{P23 TARGET AND BIOMARKER DISCOVERY FOR HEDGEHOG PATHWAY ACTIVITY IN IDIOPATHIC PULMONARY FIBROSIS IN SUPPORT OF A PHASE 2 RANDOMISED, DOUBLE-BLIND, PLACEBO-CONTROLLED STUDY TO ASSESS EFFICACY AND SAFETY OF VISMODEGIB IN IPF (ISLAND)}

${ }^{1} \mathrm{G}$ Jia, ${ }^{1} \mathrm{~S}$ Chandriani, ${ }^{1} \mathrm{AR}$ Abbas, ${ }^{1} \mathrm{D}$ DePianto, ${ }^{1} \mathrm{E}$ N'Diaye, ${ }^{1} \mathrm{M}$ Yaylaoglu, ${ }^{2} \mathrm{HR}$ Collard, ${ }^{2} \mathrm{P}$ Wolters, 'J Egen, ${ }^{3} \mathrm{~A}$ Scalori, ${ }^{3} \mathrm{~A}$ Ackrill, 'J Hou, ${ }^{3} \mathrm{~J}$ Kaminski, ${ }^{4} \mathrm{TM}$ Maher, ${ }^{1} \mathrm{~J} R$ Arron. ${ }^{1}$ Genentech, Inc., South San Francisco, USA; ${ }^{2}$ Division of Pulmonary and Critical Care Medicine, Department of Medicine, University of California, San Francisco, USA; ${ }^{3}$ Roche Ltd, Welwyn Garden City, UK; ${ }^{4}$ Royal BromptonHospital, London, UK

\subsection{6/thoraxjnl-2014-206260.173}

Objectives Idiopathic pulmonary fibrosis (IPF) is associated with aberrant expression of signalling pathways involved in embryonic development, including the Hedgehog ( $\mathrm{Hh}$ ) pathway. $\mathrm{Hh}$ can promote multiple profibrotic processes including myofibroblast differentiation, expression of extracellular matrix genes, migration, and survival. Vismodegib is a small-molecule inhibitor of the Hedgehog (Hh) signalling pathway approved for the treatment of basal cell carcinoma. We sought to evaluate the activity of Hh signalling in IPF lung tissue and identify blood biomarkers of Hh pathway activity in IPF patients.

Methods Gene expression in biopsies from IPF and control unused donor lungs, and in fibroblasts stimulated with Shh in vitro. CXCL14 protein was measured in plasma from IPF patients and from solid tumour patients treated with vismodegib in a phase $1 \mathrm{~b}$ clinical trial (NCT00968981).

Results We observed significantly increased expression levels of Hh pathway genes including SMO, PTCH2, GLI1, and GLI2 in IPF vs control lungs. To identify candidate systemic biomarkers of Hh pathway activity, we compared transcriptional data from IPF lung biopsies and fibroblasts stimulated in vitro with Shh. The gene most significantly upregulated in both datasets was CXCL14, which encodes a soluble secreted chemokine whose expression is inhibited in vitro by the addition of vismodegib. Circulating CXCL14 protein levels were significantly higher in plasma from IPF patients than controls. In solid tumour patients, circulating CXCL14 levels were significantly reduced upon treatment with vismodegib.

Conclusions We observed strong evidence for Hedgehog pathway activity in IPF lungs. CXCL14 is a systemic biomarker that could be used to identify IPF patients with increased Hh pathway activity and monitor the pharmacodynamic effects of vismodegib in IPF. The ISLAND trial is a phase 2 randomised, doubleblind, placebo-controlled study designed to evaluate the safety and efficacy of vismodegib in IPF patients. ISLAND will enrol 129 patients with IPF, randomised 2:1 to vismodegib or placebo for 60 weeks. The primary efficacy objective is to evaluate the effect of vismodegib on mean change in forced vital capacity (FVC). Secondary outcome measures include IPF and/or Hhassociated biomarkers, progression-free survival, and change in quantitative lung fibrosis on HRCT. Enrollment is expected to start in October 2014. 